\title{
Anaplastic thyroid cancer therapy: dream or reality?
}

\author{
Rossella Elisei
}

Published online: 11 October 2012

(C) Springer Science+Business Media, LLC 2012

Anaplastic thyroid cancer (ATC) is a rare thyroid malignancy representing $2 \%$ of thyroid tumors. Usually, ATC is a lethal tumor with an overall median survival time $<6$ months from the diagnosis. Anecdotic cases of long-term survivors have been reported mainly in subjects with intrathyroid tumors at the time of surgical treatment [1].

The ATC management is very challenging because patients usually present with both extensive local disease and progressive distant metastases. A wide range of conventional therapeutic approaches including thyroidectomy, radiotherapy, chemotherapy, or varying combinations of these modalities have been proposed over the years, but the outcome does not appear to be significantly modified by these strategies which are indeed largely ineffective.

This overall failure of all known methods of treating ATC is the main reason of the continuing search for novel treatment strategies. Recently, a very interesting study on the effectiveness of a combined treatment with bortezomib, a drug specifically targeting the ubiquitin-proteasome system, and MLN8054, which specifically inhibits Aurora kinases, has been reported [2]. The authors demonstrated that this combination was able to determine an antitumoral activity in 3 ATC cell lines, especially by reducing the cell growth rate and inducing cells apoptosis.

The ubiquitin-proteasome pathway is likely the most important system for the degradation of the intracellular proteins. Recently, a novel class of antitumor agents has been identified in the proteasome inhibitors which have been demonstrated to be very active against both hematological and solid tumors. Bortezomib (Velcade) has been

R. Elisei $(\bowtie)$

Department of Endocrinology and Metabolism,

University of Pisa, Pisa, Italy

e-mail: rossella.elisei@med.unipi.it officially approved by the Food and Drug Administration for the treatment of multiple myeloma and it is intensively studied in other human malignant tumors. The effect of bortezomib has been evaluated in a series of carcinoma cell lines and a proapoptotic activity on both medullary and ATC cells has been demonstrated [3].

Aurora kinases are proteins which are implicated in the regulation of multiple aspects of chromosome segregation during the mitotic phase of the cell cycle. They are overexpressed in many human thyroid tissues and their degradation at the end of the mitosis is determined by the ubiquitin-proteasome pathway. Previous studies showed that MLN8054 alone, as well as other Aurora kinase inhibitors, such as VX-680 [4] or AZD1152 [5], had proapoptotic and antiproliferative effects in ATC cell lines. These findings strongly suggest the possibility that Aurora kinase inhibitors could be used in the treatment of ATC, and, likely, in other thyroid cancer not responsive to conventional therapies.

The innovative aspect of the paper of Hoffmann and co-authors [2] recently published on this journal, is the demonstration of a synergistic effect of the combination of these two drugs which are directed against two different targets both very important for the inhibition of the cell cycle progression. The greatest interest of this study is the putative therapeutic role and clinical application.

Several other studies have been previously published with the aim to find innovative therapeutic strategies for the treatment of ATC patients. Recently, it has been reported that the antitumor and antivascular activity of CLM94, a new cyclic amide drug, was very promising when tested on ATC cells [6]. Although with different mechanisms with respect to those exerted by bortezomib and MLN8054, also CLM94 determined a significant reduction of growth proliferation and a simultaneous increase of cell apoptosis. In 
this case, it was also observed the inhibition of cell migration and invasion. Also lovastatin, a HMG-CoA inhibitor, has been demonstrated to induce apoptosis and inhibition of cell proliferation as well as of invasiveness in ATC cells [7].

Gene therapy approaches have been also explored such as the infection of ATC cells with GLV-1h153, a vaccinia virus carrying the human sodium iodine symporter (hNIS). The infected ATC cells showed hNIS-specific radiouptake which could convert the ATC cells from resistant to susceptible to radioiodine therapy [8]. A similar approach was tested by our group directly transfecting hNIS cDNA in a ATC cell line [9]. Although very fascinating, these approaches suffer from the absence of the cell machinery required for the organification of iodine. A multiple transduction of several genes (i.e., thyroperoxidase, pendrin, etc.) should be planned to overcome this problem.

The major concern about these studies is that only few of them have been reproduced in vivo, mostly performed on ATC cell lines xenografted mice. Although it is undoubted that preclinical in vitro studies are fundamental for the identification of promising new therapeutic approaches, the question of whether it is really possible to transfer the in vitro results to in vivo studies and finally in phase I or II clinical trials is worrying the experts. However, the recent initiation of a phase I/II study with the combined use of bortezomib and a Aurora kinase inhibitor in multiple myeloma is of extreme interest and can prelude a similar study in ATC affected patients.

The other very important concern is the dependence of the results on the individual cell line which could reflect an individual sensitivity of tumor cells to the two drugs. This concern could likely be eliminated if we could identify any molecular marker (i.e., oncogene mutations, specific protein expression, etc.) or morphological feature able to distinguish "responders" from "non responders patients".

While these new drugs and their therapeutic strategies are still under a preclinical phase of investigation, other drugs, which are in advanced experimental phases, deserve to be further explored. Combretastatin-A4, a novel vascular targeting agent able to block the neoangiogenesis, has been recently tested in an international multicentric randomized clinical trial exploring its effect on ATC patients. The study compared one group of patients treated with cisplatinum and paclitaxel and one treated with the same chemiotherapic agents plus combretastatin-A4 [10]. The results of this phase II study were very encouraging and it would be desirable that a phase III study would be planned in the next future.

ATC with a BRAF mutation might obtain clinical benefit from treatment with sorafenib which has been already approved for the treatment of advanced renal and epatocarcinoma. A phase II study on the clinical benefits obtained in patients with metastatic and advanced thyroid carcinoma refractory to iodine treatment has been published [11]. At the present, the results of an international, randomized phase III study are under elaboration.

In conclusion, ATC are killer tumors and, unfortunately, there are no effective therapies. New therapeutic strategies based on new molecular approaches such as that explored by Hoffmann et al., are desirable. In particular, it would be of interest to confirm these results in in vivo studies. A significant improvement of our knowledge on the tumoral transformation leading from a normal follicular cell to a very aggressive ATC cell is also needed.

\section{References}

1. S.M. Lim, S.J. Shin, W.Y. Chung, C.S. Park, K.H. Nam, S.W. Kang, K.C. Keum, J.H. Kim, J.Y. Cho, Y.K. Hong, B.C. Cho, Treatment outcome of patients with anaplastic thyroid cancer: a single center experience. Yonsei Med. J. 53(2), 352-357 (2012)

2. A. Wunderlich, S. Roth, A. Ramaswamy, B.H. Greene, C. Brendel, U. Hinterseher, D.K. Bartsch, S. Hoffmann, Combined inhibition of cellular pathways as a future therapeutic option in fatal anaplastic thyroid cancer. Endocrine (2012). doi:10.1007/s 12020-012-9665-4

3. A. Wunderlich, T. Arndt, M. Fischer, S. Roth, A. Ramaswamy, B.H. Greene, C. Brendel, U. Hinterseher, D.K. Bartsch, S. Hoffmann, Targeting the proteasome as a promising therapeutic strategy in thyroid cancer. J. Surg. Oncol. 105(4), 357-364 (2012)

4. Y. Arlot-Bonnemains, E. Baldini, B. Martin, J.G. Delcros, M. Toller, F. Curcio, F.S. Ambesi-Impiombato, M. D'Armiento, S. Ulisse, Effects of the Aurora kinase inhibitor VX-680 on anaplastic thyroid cancer-derived cell lines. Endocr. Relat. Cancer 15(2), 559-568 (2008)

5. S. Libertini, A. Abagnale, C. Passaro, G. Botta, S. Barbato, P. Chieffi, G. Portella, AZD1152 negatively affects the growth of anaplastic thyroid carcinoma cells and enhances the effects of oncolytic virus d1922-947. Endocr. Relat. Cancer 18(1), 129-141 (2011)

6. A. Antonelli, G. Bocci, C. La Motta, S.M. Ferrari, P. Fallahi, I. Ruffilli, A. Di Domenicantonio, A. Fioravanti, S. Sartini, M. Minuto, S. Piaggi, A. Corti, G. Ali, T. Di Desidero, P. Berti, G. Fontanini, R. Danesi, F. Da Settimo, P. Miccoli, CLM94, a novel cyclic amide with anti-VEGFR-2 and antiangiogenic properties, is active against primary anaplastic thyroid cancer in vitro and in vivo. J. Clin. Endocrinol. Metab. 97(4), E528-E536 (2012)

7. W.B. Zhong, S.P. Hsu, P.Y. Ho, Y.C. Liang, T.C. Chang, W.S. Lee, Lovastatin inhibits proliferation of anaplastic thyroid cancer cells through up-regulation of $\mathrm{p} 27$ by interfering with the Rho/ROCK-mediated pathway. Biochem. Pharmacol. 82(11), 1663-1672 (2011)

8. S. Gholami, D. Haddad, C.H. Chen, N.G. Chen, Q. Zhang, P.B. Zanzonico, A.A. Szalay, Y. Fong, Novel therapy for anaplastic thyroid carcinoma cells using an oncolytic vaccinia virus carrying the human sodium iodide symporter. Surgery 150(6), 1040-1047 (2011)

9. R. Elisei, A. Vivaldi, R. Ciampi, P. Faviana, F. Basolo, F. Santini, C. Traino, F. Pacini, A. Pinchera, Treatment with drugs able to reduce iodine efflux significantly increases the intracellular retention time in thyroid cancer cells stably transfected with sodium iodide symporter complementary deoxyribonucleic acid. J. Clin. Endocrinol. Metab. 91(6), 2389-2395 (2006) 
10. R. Elisei, J. Sosa, B. Jarzab et al., Randomized phase $2 / 3$ trial of a tumor vascular disrupting agent fosbretabulin (CA4P) with carboplatin and paclitaxel in anaplastic thyroid cancer (ATC). ITC Abstract Book (2010)
11. V. Gupta-Abramson, A.B. Troxel, A. Nellore, K. Puttaswamy, M. Redlinger, K. Ransone, S.J. Mandel, K.T. Flaherty, L.A. Loevner, P.J. O'Dwyer, M.S. Brose, Phase II trial of sorafenib in advanced thyroid cancer. J. Clin. Oncol. 26(29), 4714-4719 (2008) 